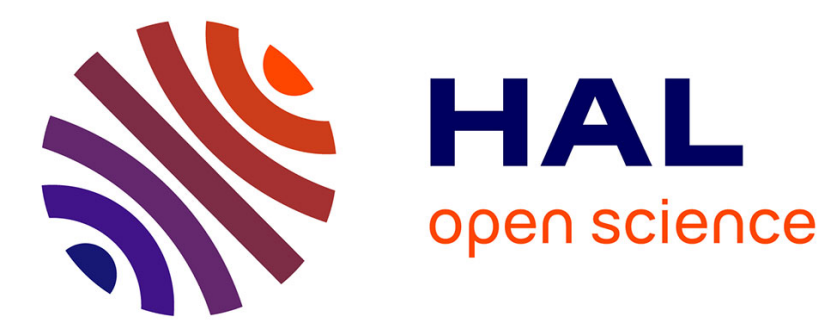

\title{
Raters' reliability in clone benchmarks construction
}

Alan Charpentier, Jean-Rémy Falleri, Floréal Morandat, Elyas Ben Hadj

Yahia, Laurent Réveillère

\section{To cite this version:}

Alan Charpentier, Jean-Rémy Falleri, Floréal Morandat, Elyas Ben Hadj Yahia, Laurent Réveillère. Raters' reliability in clone benchmarks construction. Empirical Software Engineering, 2017, 22 (1), pp.235-258. 10.1007/s10664-015-9419-z . hal-02182070

\section{HAL Id: hal-02182070 \\ https://hal.science/hal-02182070}

Submitted on 10 Jan 2020

HAL is a multi-disciplinary open access archive for the deposit and dissemination of scientific research documents, whether they are published or not. The documents may come from teaching and research institutions in France or abroad, or from public or private research centers.
L'archive ouverte pluridisciplinaire HAL, est destinée au dépôt et à la diffusion de documents scientifiques de niveau recherche, publiés ou non, émanant des établissements d'enseignement et de recherche français ou étrangers, des laboratoires publics ou privés. 


\title{
Raters' Reliability in Clone Benchmarks Construction
}

\author{
Alan Charpentier · Jean-Rémy Falleri · Floréal \\ Morandat · Elyas Ben Hadj Yahia · Laurent \\ Réveillère
}

Received: date / Accepted: date

\begin{abstract}
Cloned code often complicates code maintenance and evolution and must therefore be effectively detected. One of the biggest challenges for clone detectors is to reduce the amount of irrelevant clones they found, called false positives. Several benchmarks of true and false positive clones have been introduced, enabling tool developers to compare, assess and fine-tune their tools. Manual inspection of clone candidates is performed by raters that do not have expertise on the underlying code. This way of building benchmarks might be unreliable when considering context-dependent clones i.e., clones valid for a specific purpose. Our goal is to investigate the reliability of rater judgments about context-dependent clones. We randomly select about 600 clones from two projects and ask several raters, including experts of the projects, to manually classify these clones. We observe that judgments of non expert raters are not always repeatable. We also observe that they seldomly agree with each others and with the expert. Finally, we find that the project and the fact that a clone is a true or false positive might have an influence on the agreement between the expert and non experts. Therefore, using non experts to produce clone benchmarks could be unreliable.
\end{abstract}

Keywords Duplication $\cdot$ Code clone $\cdot$ Empirical study $\cdot$ Software metrics

\author{
Alan Charpentier \\ University of Bordeaux, LaBRI, UMR 5800, F-33400, Talence, France \\ Tel.: +33 (0)5-4000-3554 \\ E-mail: acharpen@labri.fr \\ Jean-Rémy Falleri \\ University of Bordeaux, LaBRI, UMR 5800, F-33400, Talence, France \\ E-mail: falleri@labri.fr \\ Floréal Morandat \\ University of Bordeaux, LaBRI, UMR 5800, F-33400, Talence, France \\ E-mail: fmoranda@labri.fr \\ Elyas Ben Hadj Yahia \\ University of Bordeaux, LaBRI, UMR 5800, F-33400, Talence, France \\ E-mail: elyas.bhy@labri.fr \\ Laurent Réveillère \\ University of Bordeaux, LaBRI, UMR 5800, F-33400, Talence, France \\ E-mail: reveillere@labri.fr
}




\section{Introduction}

Redundant code in software systems is referred as code clones in the literature. Two code fragments form a code clone if they are similar or identical [3]. Code clones can arise due to the use of so-called "copy-paste" development, in which a developer creates new code by copying existing code that is expected to have similar intent, and possibly modifying it slightly according to its new context. Duplicating code can reduce dependencies between modules and help new developers conform to the prevailing coding style. While cloning code is not always harmful to the system quality [15], it often complicates code maintenance and evolution, as fault fixes and changes must be propagated from one instance of a clone to the others. Therefore, software developers tend to keep the number of code clones as low as possible.

The desirability of identifying, and sometimes eliminating, clones has led to the development of clone detectors, which scan a code base for potential clones [9,11,13,20,24]. Several studies have used such tools to estimate the number of code clones in a software project, reporting from $5 \%$ to $23 \%$ of cloned code $[1,2,18]$. One of the biggest challenges for an effective application of clone detection in practice is detection precision. Indeed, some code clones reported by a clone detector may be viewed as false positives as they are irrelevant for the user of the detection tool. In addition, identifying false positives is made challenging simply by the amount of data that a clone analysis of a large project generates.

A traditional approach to reduce the number of false positives is to enhance the clone detection algorithm and fine-tune the detection tool. To support this tuning, several benchmarks of clones have been proposed [3,17,27], enabling tool developers to assess the results of their tools. Recently, machine learning techniques have been introduced to automatically classify generated clones in true or false clones based on feedback from the user of the tool [30]. These approaches rely on a set of clones that have already been rated as true or false positives. Producing such a set of clones always requires a human judgment. Manual inspection of clone candidates is usually performed by students or researchers who may have no or few expertise on the underlying software projects.

Clone candidates from existing clone benchmarks are usually classified without any specific purpose in mind. We call such benchmarks context-free as there is no relation between their usage and the way they are defined. As a consequence, one could ask to which extent the present way of building clone benchmarks is reliable when considering contextdependent clones i.e., clones classified as useful with respect to a specific purpose.

In this paper, we investigate the reliability of rater judgments about context-dependent clones. Our goal is to bring out several guidelines to ease the construction of contextdependent clones benchmarks. In this experiment, we address both refactoring and coevolution activities. We consider two software projects and for each of them a set of four raters to judge the results produced by a clone detector. Among the raters, three are external raters with no or few preliminary knowledge about the project and one is an expert, one of the main developers of the project. Expert's judgment is very important for a contextdependent clone benchmark because the clones identified by a clone detector have to be useful for her. Hence, the two experts are oracles deciding whether a clone is a true or false positive, according to a given context. Finally, we use the answers of the raters to discuss the reliability of rater judgments.

We show that using external raters to build context-dependent clone benchmarks may be unreliable. We also show that the project being studied may have a significant impact on the agreement between external raters and experts. Additionally, we find that true positives clones (as judged by the experts) seem to be significantly harder to judge by external 
raters. Based on our findings, we recommend to use several external raters to build contextdependent clone benchmarks and to involve experts to validate true positives.

The structure of this paper is as follows. Section 2 describes related work. Section 3 introduces the issue of detection precision. Next, we describe our empirical study methodology in section 4. Section 5 presents the findings of our empirical study; it highlights a number of research questions and their answers. We discuss the threats to the validity of our study in section 6. We conclude and mention future work in section 7.

\section{Related Work}

In this section, we provide some background about clone benchmarks and clone rater reliability.

\subsection{Clone benchmarks}

Bellon et al. provide a benchmark of clones to compare and evaluate clone detection tools [3]. Six researchers helped Bellon to construct the benchmark. Each of them applied his own clone detector on eight large $\mathrm{C}$ and Java programs and provided the found clones to Bellon. Bellon classified alone $2 \%$ of the 325,935 submitted clones and built a reference corpus by retaining only clones he judged as true positives. Clone detectors' results are compared to the reference corpus, and assessed using the traditional information retrieval measures: precision and recall. This benchmark is largely used by the research community $[6,16,23$, 26,29].

Krutz and Le built a set of function-level clones to help the evaluation of clone detection tools [17]. They asked seven persons to judge 1,536 randomly drawn function pairs from three open source programs: Apache, Python and PostgreSQL. Three are experts who have research experience with code clones and four are students with programming expertise but no prior experience with clones. Students were asked to read related papers beforehand to familiarize with the notion of clones. Their process is more rigorous than the one used by Bellon et al. [3] but the size of their benchmark is significantly smaller, which might impact its usefulness.

Svajlenko et al. provide a function-level benchmark of inter-project clones [27], called BigCloneBench. This benchmark is built independently of clone detection tools, by using a search-based approach. Hence, it is not limited to the clones that tools are able to identify. This benchmark aims at containing clones related to ten functionalities (such as bubble sort or zip decompression). For a candidate clone, several raters manually check if it is a true or false positive clone by deciding whether or not related code fragments implement the same functionality. The judges are provided a clear specification of what implementing the functionality requires. This benchmark differs from the others on clone validation. It is the only one considering context-dependent clone. Context matches a functionality.

Roy and Cordy propose a mutation / injection based framework for empirically evaluating clone detection tools [25]. The framework has two main phases. First, mutated code fragments are created from their proposed editing taxonomy for cloning. Second, these mutated code fragments are injected into original code base. Then, precision and recall values are computed and used to evaluate individual tool or to compare different tools. Their framework does not rely on clone validation since the clones are automatically created. Thus, it 
avoids the validation issues. However as mentioned in the paper, their editing taxonomy cannot guarantee to create clones for a particular task, such as refactoring or co-evolution.

In all the benchmarks we present above, no raters are experts on the programs used to build the clone benchmarks ${ }^{1}$. While this way of building benchmarks is valid for contextfree clones, one can ask whether it is still correct when considering context-dependent clones. In our study, we investigate this potential threat to validity of the construction of context-dependent clone benchmarks and assess its impact.

\subsection{Clone raters reliability}

Walenstein et al. use the corpus provided by Bellon et al. [3] to investigate the level of agreement among raters [28], who are researchers. They claim that past reports of relevance and precision for clone detectors have to be interpreted with caution because of the problem of inter-rater reliability. Additionally, they find that the agreement among raters strongly depends on the projects being studied and on the question asked to raters. In this article, we also observe that the choice of the project has a significant influence on the agreement.

Kapser et al. perform a study to assess agreement among researchers when classifying potential clones as true or false positives [14]. They use CCFinder [13] to select 20 candidate clones from the PostgreSQL source code. They observe that only $50 \%$ of candidate clones are classified in the same way by more than $80 \%$ of the raters. They highlight the importance of reporting the criterion used for clone judgment in order to make possible the comparison with previous works. In this article, we also observe that raters can have different judgments about clones.

Mende et al. propose an approach to support the grow-and-prune model [8] in the evolution of software projects by using clone detection [22]. They evaluate their technique by measuring recall and precision with respect to a benchmark. They consider multiple raters to increase the confidence of the judgments. The raters, five researchers and four graduate students in computer science, judge the same pairs of functions. A concrete scenario is given to the raters to help them decide whether or not two functions are similar. Authors find a reasonable agreement among the raters. This result is different from the observation we make in this article. However, the work of Mende et al. considers function clones, and our work is about all types of clones. This could have an influence on the agreement of raters. Additionally, the clones of Mende et al. were not validated by an expert of the analyzed code. Such an expert may have a different judgment about these clones.

In a previous work [5], we analyzed the reliability of the reference clones contained in Bellon's benchmark. We showed that there are debatable clones in this benchmark, and that it might alter the values of precision and recall computed using the benchmark. In this article we go beyond this previous work. First, raters are asked to judge context-dependent clones, either for refactoring or co-evolution activity. Second, we add experts of the underlying code in the experiment. We found that agreeing with an expert is hard, especially for true positive clones.

\section{Research Questions}

Manual inspection of clone candidates in existing benchmarks is usually performed by students or researchers who are not experts of the underlying software projects. Additionally,

\footnotetext{
1 These studies mentioned no information about any raters' expertise of the analyzed code.
} 
these raters generally do not receive any training before judging the clones. Nevertheless, since these benchmarks are context-free, one can ask to which extent this way of building clone benchmarks is reliable when considering context-dependent clones?

In this study, we explore the reliability of rater judgments about context-dependent clones. We focus our research on two main points. First we investigate the repeatability of raters' answers. We wonder if, when a rater is presented several times the same clones, she will always judge the clone consistently. Second, we explore inter-rater reliability. More precisely, we evaluate if several raters judge the clones identically. We include an expert of the code in the set of raters. Reflecting the judgment of an expert is very important for a context-dependent clone benchmark because the clones reported by a clone detector must be useful for her. Therefore we evaluate if external raters can judge clones in the same way as an expert of the code. Finally we also evaluate if there are some clones for which it is easier to reach an agreement between external raters and experts. To sum-up, we investigate the following research questions.

Repeatability of rater answers

RQ1. Are rater answers consistent over time?

As previously explained, raters are usually not trained before judging clones. This could be a threat to the repeatability of their answers, since there is a chance that they can be subject to a learning effect when rating clones. In this research question, we want to assess if a rater would always judge the same clone in the same way. We also want to refine this question by distinguishing experts from external raters. If the judgments of the clones are not repeatable, it could be a sign of a learning effect.

Inter-rater reliability

RQ2. Do external raters agree with each others, and also with the expert of a project?

In this research question, we want to assess if several raters judge the clones identically. First of all, we want to evaluate to which extent several external raters agree with each others. Then we want to evaluate if the external raters agree with the experts. By answering this research question, we know if it is reliable to build context-dependent clone benchmarks by using external raters instead of experts.

RQ3. What are the characteristics that influence the agreement between experts and external raters?

In this research question, we want to evaluate if some characteristics make easier for external raters to have the same judgment as the expert. We investigate this question because if some characteristics are shown to make the clone very easy to judge in the same way as the expert by the external raters, we can build reliable context-dependent clone benchmarks by including clones having these characteristics, without the need of an expert.

We investigate two types of characteristics: clone based characteristics and project and expert based characteristics.

The clone-based characteristics are as follows:

- Type: is it easier to judge Type-2 or Type-3 clones (as defined by Bellon et al. [3])? We do not consider Type-1 clones as they are less debatable. 
- Size: is it easier to judge clones having big fragments than clones having small fragments?

- Distance: is it easier to judge clones in completely different files or in the same file?

The project and expert based characteristics are as follows:

- Project: is it easier to judge clones from one project rather than another one?

- False/true positives: is it easier to judge true or false positive clones, as judged by the expert?

In the remainder of the paper, clones refer to context-dependent clones.

\section{Experimental setup}

We describe in this section the empirical study we conduct to answer our research questions and investigate the accuracy of user judgments about clones. All data used and collected during this study is available online ${ }^{2}$.

\subsection{Overall approach}

Our experiment consists in drawing randomly a set of clones from selected projects and present them to four raters, including an expert of each selected project. The raters are asked to judge the clones, through a web interface. The two code fragments of each clone are displayed to the rater and she is asked to rate yes in case of a true clone, no in case of a false clone, and unknown otherwise, i.e., if there is no obvious answer. Finally we use a statistical analysis of the produced data to answer our research questions. This analysis is presented in section 5 .

\subsection{Subjects and objects}

We describe in this section the subjects and objects of our experiment. First we describe the software projects on which we compute the clones. Then we explain which clone detector was used, and how it has been configured. Finally, we present the raters who participated in this experiment.

\subsubsection{Software projects}

Our experiment requires an expert for each project under study. Thus, we select two projects developed by people from our research group: Fast $\mathrm{R}^{3}$ and GumTree ${ }^{4}$. Both projects use Java as the main language. FastR [12] is an open-source efficient implementation of the $\mathrm{R}$ statistical language [10]. GumTree [7] is an open-source abstract syntax tree based code diff tool.

To prevent the clone detector to search clones in locations that are irrelevant for the user, the code base is pre-processed according to the advice of the expert of each project. First,

\footnotetext{
2 http://www.labri.fr/perso/acharpen/ese15/materials.zip

3 https://github.com/allr/fastr/tree/v0.168

4 https://github.com/jrfaller/gumtree/tree/v1.0.0
} 
Table 1 Software projects' description

\begin{tabular}{lccc}
\hline Project & Java Files & Java LOC & \# Clones \\
\hline FastR & 343 & 54,511 & 49,911 \\
GumTree & 77 & 4,750 & 216 \\
\hline
\end{tabular}

files that are automatically generated and never modified manually are discarded. Second, files corresponding to tests and examples are also discarded. Indeed, such code is usually maintained in a different way than the code from the core. At the end, we stripped out blank lines and comments in all remaining files. Table 1 summarizes the number of files and the number of lines of code (LOC) in Java we obtain for each project. GumTree is a small-sized project and FastR is a medium-sized project. The small size of GumTree allows to manually investigate all clones a clone detector could report.

\subsubsection{Clone detector}

Various clone detectors have been introduced to identify clones from source code. All of them can be tuned using plenty of parameters. Therefore, the choice of a specific clone detector and its configuration parameters may have a huge impact on the list of computed clones. In this study, we rely on the work of Wang et al. [29] to minimize the impact of this choice. They introduce an approach to find suitable configurations for empirical studies. We choose a configuration that maximizes recall because we want an overview of all the clones present in the selected projects.

We choose iClones [9] as the clone detector for two reasons. First, it is easily available for replication purposes. Second, it is the only reputed Type- 3 clone detector in the literature that is compatible with Java Generics, and both selected projects use this feature a lot. iClones has two parameters: minblock, the minimum length of identical token sequences that are used to merge near-miss clones, and minclone, the minimum length of clones measured in tokens. As recommended by Wang et al. to maximize recall for Java projects, we set minblock to 6 and minclone to 26 .

\subsubsection{Clone raters}

The four raters participating to this experiment are authors of the paper. The fifth author, namely Alan Charpentier, does not rate clones. He is responsible for running a fair experiment to answer predefined research questions. He was the only one to know the research questions during the survey.

All participants have an extensive Java programming experience and all are more than familiar with the notion of clones. For each project, we consider one expert and three external raters. For FastR, the expert is Floréal Morandat, one of the main developer of this project. For GumTree, the expert is Jean-Rémy Falleri, its official maintainer. Non expert raters are external raters and denoted by rater 1 , rater 2 and rater 3 in the remainder of the paper.

\subsection{Clone selection}

For each project, we run iClones to compute a list of clones. Table 1 reports the number of clones (only Type-2 and Type-3) identified by iClones in the two selected projects, using 


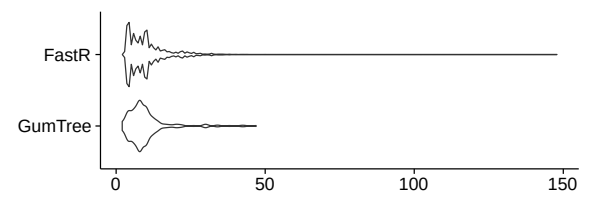

(a) Size characteristic

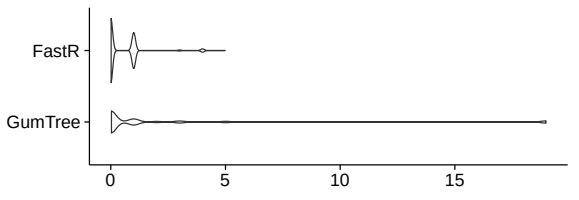

(b) Distance characteristic

Fig. 1 Distribution of clone sizes and distances in FastR and GumTree.

the configuration presented above. We remind that in $\mathbf{R Q 3}$, we want to explore if there are factors that have an effect on the judgment of raters. Some factors, namely type, size and distance are information computed from the clones. We use the same definition of type as Bellon et al. [3]. Clone size is defined as the number of lines of code of the two code fragments involved laid end to end. Clone distance represents how close are these two code fragments in the file system tree. If the two fragments are in the same file, the distance is 0 . If they belong to different files within the same directory, the distance is 1 . In any other cases, the distance is 1 plus the minimum number of hops to reach one file from the other.

To ensure having at least some representatives of each characteristic, clones are randomly drawn according to their characteristic. Concerning the type factor, once Type-1 removed (see RQ3), there are two natural clusters: Type-2 and Type-3. However, there is no natural definitions of clusters for size and distance. By performing an exploratory analysis in our projects of both the clone sizes and distances distribution (figure 1), we noticed that these distributions are right skewed with a very long tail. Therefore we choose to separate the clones in two clusters, the ones from the head of the distribution, and the ones from the tail. To avoid the bias of selecting a threshold, we use an automatic technique to create the clusters for size and distance. Additionally, we want this technique to be deterministic so that researchers can reproduce our results. To fulfill these requirements we use the neural gas data-mining algorithm [21] which is a generalization of k-means that produces stable clusters. On our corpus, tens of runs of neural gas produce clusters without any significant difference. Finally we end up with four clusters. Two are from the size factor: $\Sigma$-Big and $\Sigma$-Small, and two from the distance factor: $\Delta$-Close and $\Delta$-Far. The computed threshold to separate the two clusters of the size is 17 (resp. 18) for FastR (resp. GumTree). In other words, clones in FastR (resp. GumTree) having a size lower or equal to 17 lines (resp. 18) are in $\Sigma$-Small, while others are in $\Sigma$-Big. The thresholds for the distance are 0 for FastR and 5 for GumTree.

Based on our previous experience of rating clones, the number of clones to rate by each participant has been limited to 600 . Since we have six clusters, we draw at random 50 clones in each cluster for both projects. However, if a cluster has less than 50 clones, we select all clones of this cluster. This case only happens for GumTree in two clusters that do not contain enough elements: $\Sigma$-Big and $\Delta$-Far with both 17 clones. As a result, we obtain a set of 300 clones for FastR and another of 234 clones for GumTree, amounting to 534 clones to rate.

In addition to the type, size and distance factors, we also evaluate the influence of the expert opinion on the clones. Therefore we also compute the union of all clusters for each project. We call this union general and we use this sample to evaluate the effect of the two aforementioned factors. Since cluster sampling is likely to produce biased samples when clusters are joined, we plot the distribution of the clones of our samples against the clones of the whole project in figure 2. As reported in table 1, FastR contains 49,911 clones and GumTree 216. For GumTree, we can see that clusters $\Sigma$-Big and $\Delta$-Far have very few ele- 


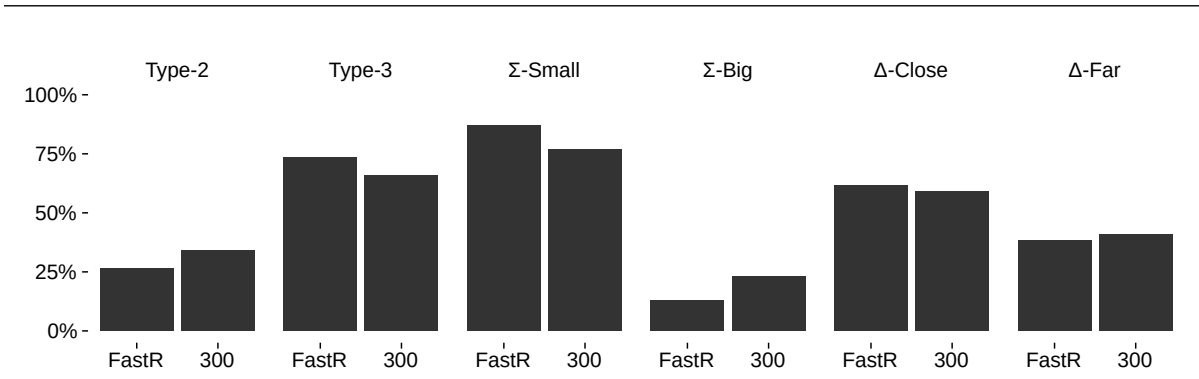

(a) FastR and our sample of 300 clones.

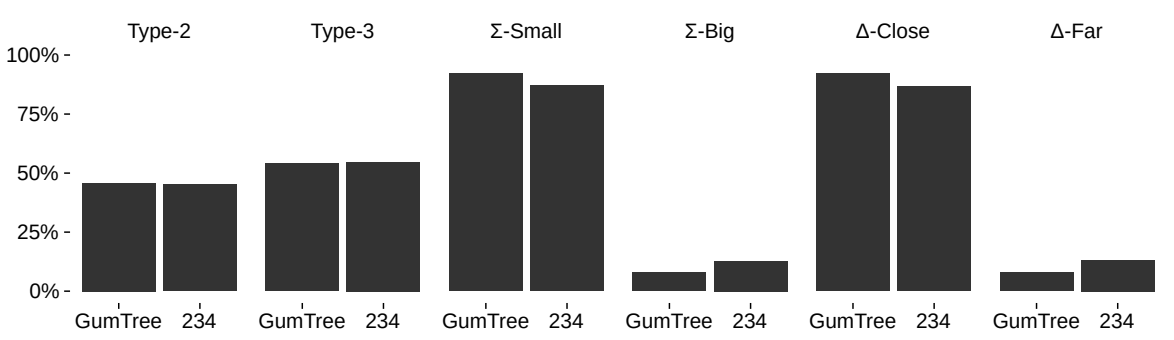

(b) GumTree and our sample of 234 clones.

Fig. 2 Distribution of clones in each cluster for each project and its associated sample.

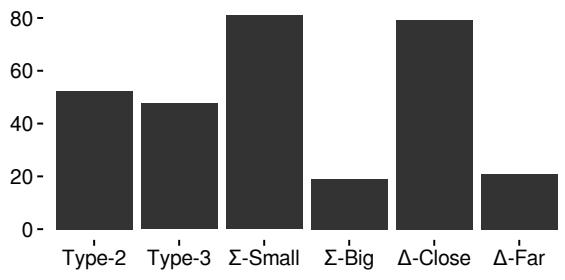

Fig. 3 Distribution in each cluster of the 65 duplicates in GumTree sample.

ments (less than 17\%, corresponding to 50/300). Additionally, one can see that each project and its associated sample have a similar distribution of clones according to the six clusters.

Since clones of each cluster are randomly selected from the whole set of clones identified by iClones for a project, duplicates may appear. In particular, since the number of clones drawn in GumTree was greater than the total number of clones, duplicate clones were mandatory. This was done on purpose to evaluate the repeatability of rater judgments as stated in RQ2. One author of the paper, Alan Charpentier, has checked for duplicates in the set of 534 clones. As a result, there are 65 duplicates in the 234 clones from GumTree and 1 in the 300 clones from FastR. In other words, there are in fact 163 unique clones for GumTree and 299 for FastR. As previously explained, this author did not warn the other raters of the presence of duplicates in the set of clones to rate. Thus, only Alan Charpentier knew that there were duplicates. Figure 3 shows the distribution of the duplicates from GumTree in the clusters. One can notice that this distribution is similar to the general distribution shown in figure 2 . 


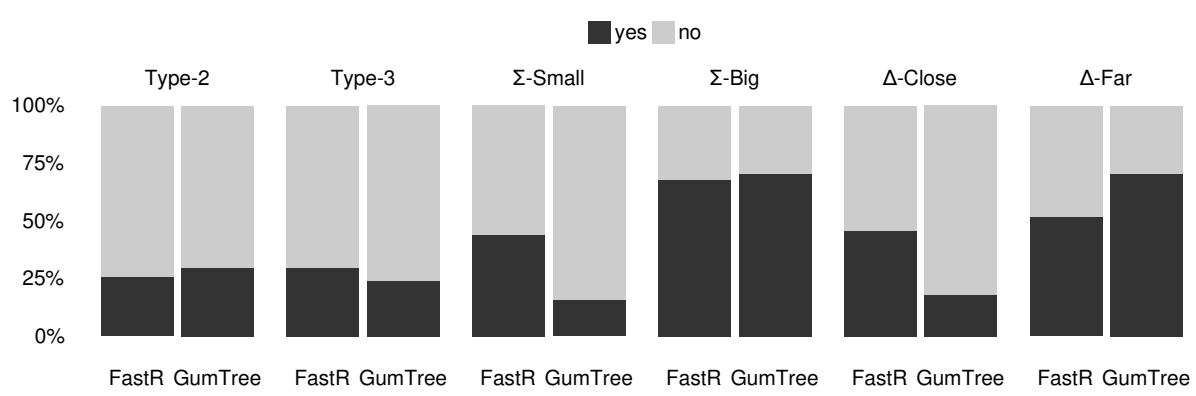

Fig. 4 Ratios of yes and no answers given by the expert on the clones of each project.

\subsection{Data collection}

In this section, we first describe the procedure we use to gather judgments from raters and then we present the raw results we obtained.

\subsubsection{Procedure}

Answers are collected through a web interface. This interface displays the two files involved in the clone, highlights in yellow the fragments belonging to the clone in each file, and shows a text diff in orange inside both fragments. To avoid rater fatigue, the web interface is able to save and restore the work session, and to go back to already processed clones to change the answer. It therefore enables the raters to process their clones taking as much time as they need. Raters were able to complete the survey in several days, splitting their work as they wished. The order of the clones has been randomized, and they are presented to each rater in the same order. The question we ask to the participants is the following: "Is this clone useful for refactoring or co-evolution purpose". To avoid to forcing the rating of clones, the external raters were allowed to answer unknown to the question, meaning that they do not have a judgment. The participants were forbidden to exchange about the clones and the experiment until it was completed by everybody. During the clone rating process, only Alan Charpentier had knowledge of the research questions and how the clones were selected. Other raters did not know the research questions.

\subsubsection{Results}

Every participant went through the 534 clones. For each project, additionally to the answers from the expert and the external raters, we also compute a majority vote that aggregates the votes from the external raters. To compute this majority vote we use the following rules. If two external raters have answered yes (respectively no) the majority vote is yes (respectively no). In any other cases, the majority vote is unknown.

Results of this experiment for the experts of each project are shown in figure 4. One can see ratios of true and false positive clones found by the expert of each system. For each project, ratios are given for each of the six clusters as well as for all the clones of the project (denoted by general as explained in section 4.3). Overall, the FastR expert finds a slightly greater number of true clones than the GumTree expert, but the mean ratio of true clones is in the same range for both projects. 


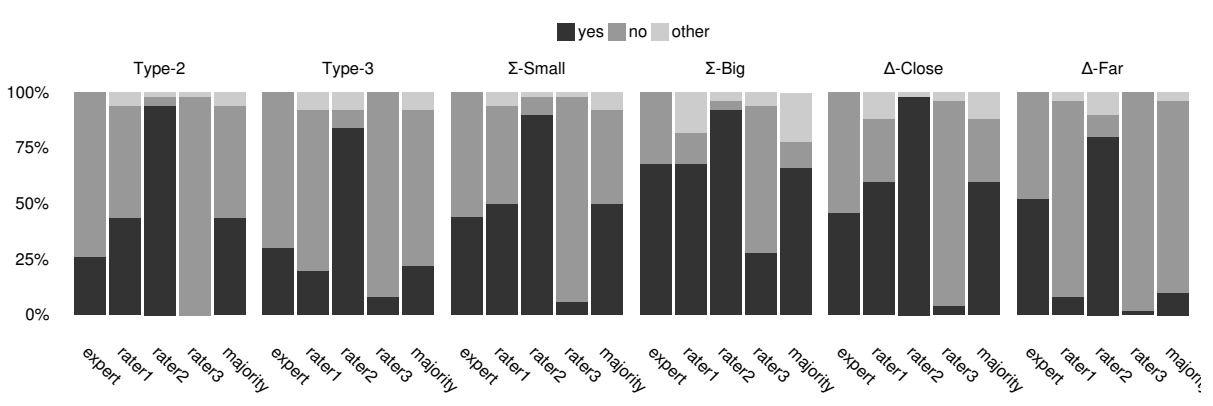

(a) FastR sample

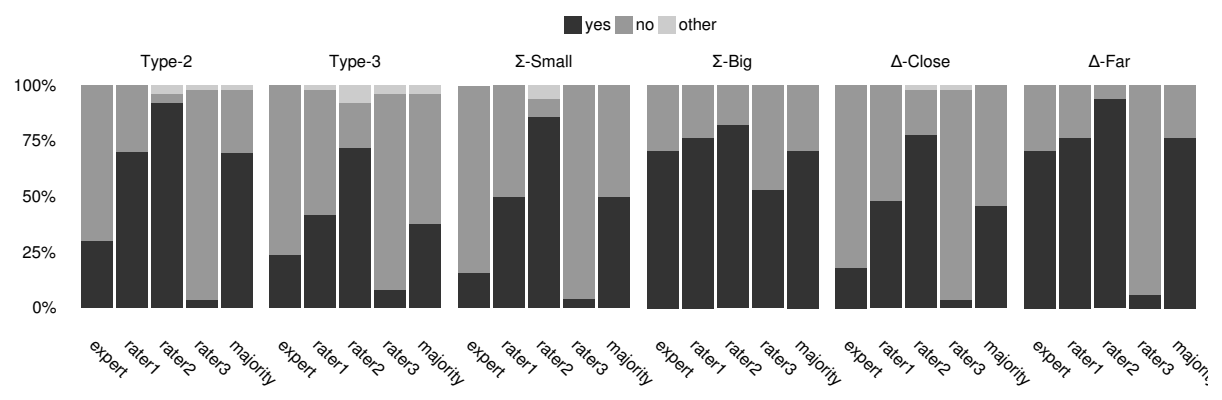

(b) GumTree sample

Fig. 5 Ratios of yes, no and unknown answers given by the experts, the external raters and the majority for the clones of each project.

Both experts seem to agree that the $\Sigma$-Big cluster contains mostly true positive clones. Additionally, the $\Delta$-Far cluster seems to contain also a higher ratio of true clones than the other clusters for both experts. All other clusters contain more false positives than true positives. In this study, we consider expert answers as references, that is to say when an expert answers yes (respectively no) we have a true positive or true clone (respectively a false negative or false clone).

The external raters have different behaviors. Their answers are shown in figure 5. For the FastR project, rater 1 and rater 3 are pessimistic about the clones while rater 2 is optimistic. The $\Sigma$-Big and the $\Delta$-Close clusters are the only ones in which a majority of external raters finds more true positives than false positives. For the GumTree project, raters 1 and 2 are much more optimistic than rater 3 . The $\Sigma$-Big cluster is the only one in which all raters find a majority of true clones. In this project, the majority vote has a significant ratio of true positives in the Type-2 and $\Delta$-Far clusters.

\section{Results and discussion}

In this section, we first present the results of our study according to each research question and provide some guidelines to make the construction of context-dependent clone benchmarks more reliable. We provide the feedback from the raters collected in a discussion session organized after the experiment. 


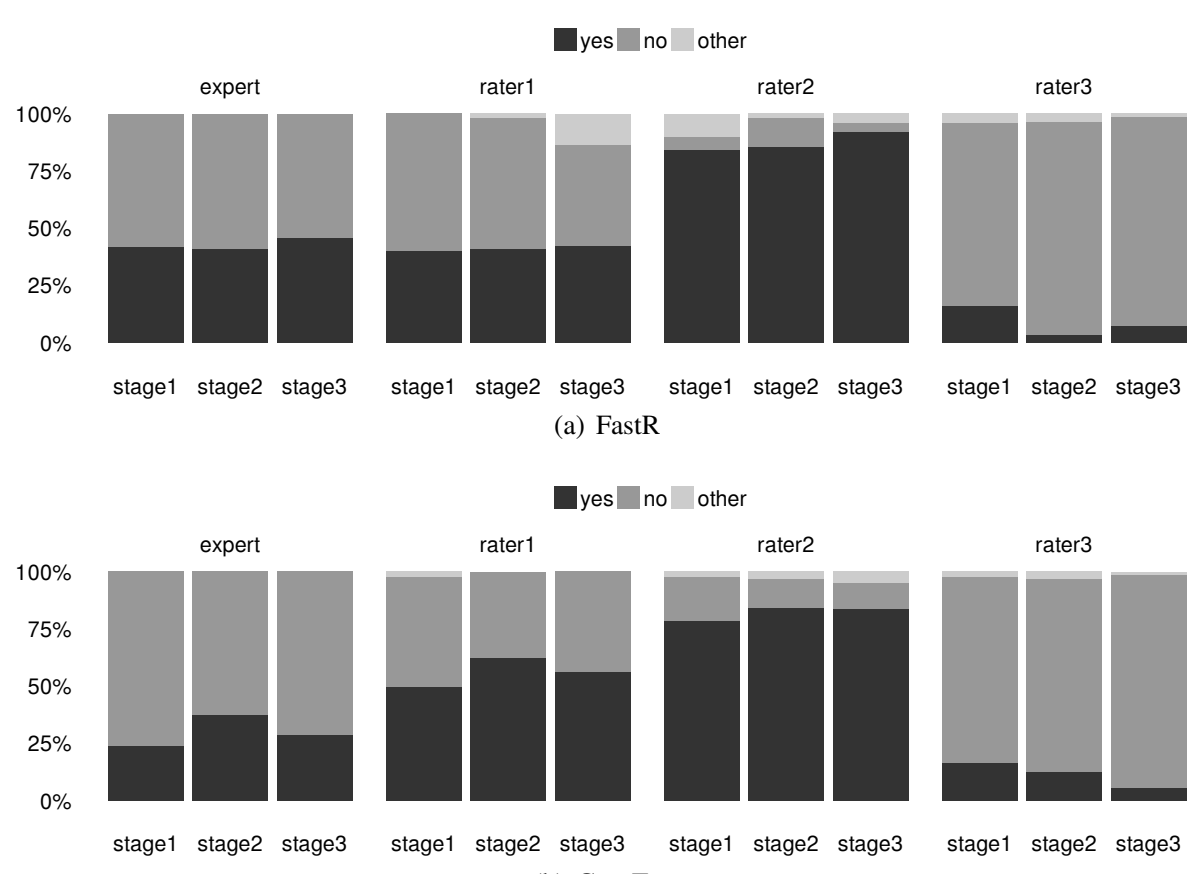

(b) GumTree

Fig. 6 Rater answers over time (stage 1: first third of the clones, stage 2: second third and stage 3: last third).

\subsection{Repeatability of raters answers (RQ1)}

The first research question is related to the consistency of rater answers. By consistent, we mean that for a given rater, the same clone always receive the same judgment. To evaluate this phenomenon we assess if there is a change of behavior of raters across time. We postulate that since the order in which clones are presented to the raters is randomized, the ratio of yes, no and unknown answers should stay stable during all the experiment. If it is not the case, it means that a learning effect or a fatigue effect biased the answers of the raters. To quantify this phenomenon we split the clones of each project in three sets. Stage 1 is the first third of the clones rated clones, stage 2 (resp. 3) is the second (resp third) third of clones. As shown in figure 6, there is no significant variation of the ratio between the stages which means that the behavior of raters remains stable across time. However, this does not indicate that a rater will judge consistently the same clone.

To evaluate the consistency of rater answers, we use the 59 duplicates contained in GumTree clones. First we investigate if raters can change between a yes, no or unknown answer for a same clone. For each rater, we compute the ratio of these clones for which the rater did not give the same judgment. Results depicted in table 2 show that the behavior of the expert is different from the one of the external raters. The expert gives very consistent answers and only 1 duplicate received a different judgment, which is negligible. While for external raters, the number of inconsistently rated clones varies from about $5 \%$ to about $20 \%$ of the rated duplicates, which is significant. We investigated if there are some trends in the way inconsistent answers evolve across time, however there are not enough inconsistent duplicates to conclude. As a general conclusion on inconsistencies, they may be attributed 
Table 2 Inconsistent answers for the 59 duplications in GumTree.

\begin{tabular}{lcc}
\hline Rater & $\#$ & $\%$ \\
\hline expert & 1 & 1.7 \\
rater1 & 11 & 18.6 \\
rater2 & 3 & 5.1 \\
rater3 & 7 & 11.9 \\
\hline
\end{tabular}

to some kind of learning effect, hence a training session would be beneficial for external raters.

\subsection{Inter-rater reliability}

In this section, we investigate the inter-rater reliability of clone judgments. First, we evaluate if several external raters give or not the same judgments about the same clones. Then, we investigate if the external raters give the same judgments as an expert of the project. Finally, we investigate if there are factors that allow external raters to better approximate the judgment of experts.

\subsubsection{Agreement among raters $(R Q 2)$}

Firstly, we evaluate the agreement between the external raters using Fleiss' Kappa. The result of this measure of an agreement in a group of person is shown in the first line of table 3. This statistic is interpreted using the thresholds provided by Landis and Koch [19] shown in table 4 . We can see that in both projects, there is no agreement among the external raters, meaning that they give divergent judgments about the same clones. It therefore seems that judging clones is a very subjective task. Secondly, we evaluate the agreement between each external rater and the expert of both projects. Results are shown in lines 2 to 4 of table 3 . Finally, we evaluate the agreement between the majority vote of external raters (as defined in section 4.4) and the expert of both projects. Line 5 shows the values obtained using Cohen's Kappa to measure the agreement. We observe a different behavior in the two projects. It seems easier for the external raters to agree with the expert in GumTree than in FastR. We also observe that the choice of a particular external rater has a high impact on the agreement with the expert. For instance in FastR the agreement is at worse slight and at best fair, and for GumTree the agreement is at worse slight and at best moderate. Therefore, using only one external rater to judge clones is unreliable. Finally, we can see that using the majority vote of external raters instead of using a particular external rater is a good strategy for both projects. Indeed the maximum value of the Kappa statistic (bolded in table 3) is reached in this case for both projects which means that the majority always has a best agreement with the expert than any particular external rater.

\subsubsection{Factors influencing agreement between external raters and experts (RQ3)}

In this research question, we investigate if there exists factors that make it easier for external raters to agree with the expert. Since the majority vote of external raters has proven to be the best strategy in the previous section, we only consider for this research question the judgment of the majority. Figure 7 reports raw agreement between the expert and the 
Table 3 Agreement among raters.

\begin{tabular}{lcccccc}
\hline \multirow{2}{*}{\multicolumn{1}{c}{ Raters }} & \multicolumn{2}{c}{ FastR } & & \multicolumn{2}{c}{ GumTree } \\
\cline { 2 - 3 } \cline { 5 - 6 } \cline { 5 - 6 } & $\kappa$ & Agreement & & $\kappa$ & Agreement \\
\hline external raters & -0.12 & none & & -0.05 & none \\
expert and rater1 & 0.24 & fair & & 0.42 & moderate \\
expert and rater2 & 0.09 & slight & & 0.13 & slight \\
expert and rater3 & 0.16 & slight & & 0.28 & fair \\
expert and the majority & $\mathbf{0 . 2 5}$ & fair & & $\mathbf{0 . 4 4}$ & moderate \\
\hline
\end{tabular}

Table 4 Kappa statistic interpretation.

\begin{tabular}{lr}
\hline$\kappa$ & \multicolumn{1}{c}{ Interpretation } \\
\hline$\leq 0$ & No agreement \\
] $0,0.2]$ & Slight agreement \\
] $0.2,0.4]$ & Fair agreement \\
] $0.4,0.6]$ & Moderate agreement \\
] $0.6,0.8]$ & Substantial agreement \\
] $0.8,1]$ & Almost perfect agreement \\
\hline
\end{tabular}

Table 5 Number of identical and different answers between the expert and the majority.

\begin{tabular}{cccccc}
\hline & \multicolumn{2}{c}{ FastR } & & \multicolumn{2}{c}{ GumTree } \\
\cline { 2 - 3 } \cline { 5 - 6 } Characteristic & Identical & Different & & Identical & Different \\
\hline Type-2 & 26 & 24 & & 27 & 23 \\
Type-3 & 35 & 15 & & 39 & 11 \\
\hline$\Sigma$-Small & 23 & 27 & & 31 & 19 \\
$\Sigma$-Big & 33 & 17 & & 17 & 0 \\
\hline$\Delta$-Close & 26 & 24 & & 34 & 16 \\
$\Delta$-Far & 26 & 24 & & 16 & 1 \\
\hline General & 169 & 131 & 164 & 70 \\
\hline
\end{tabular}

majority for each characteristic under study. First observations tend to indicate that some characteristics have an impact on the agreement between the majority and the expert (e.g. Size or Project). In the remainder of this section, we evaluate statistically the influence of characteristics on the agreement between the expert and the majority. First, we present the effect of clone characteristics. Then, we present the effect of the factors related to the expert judgment on the clones.

Clone characteristics To evaluate the effect of clone characteristics we use two clusters for each characteristic (as explained in section 4.3). For each cluster we are interested in two numbers: the numbers of identical and different answers with the expert. Therefore, for each characteristic, we construct a $2 \times 2$ contingency table as shown in table 5. Our null hypothesis is that the number of identical/different answers with the expert is independent of each characteristic. Our alternative is that this number is not independent. We use the chi-square test to assess our hypothesis. We apply Yates' continuity correction to chi-square computation when the value in a cell is too small i.e., lower than 5. Additionally, we compute the effect size of each characteristic using Cramer's V that is interpreted as shown in table 6. 


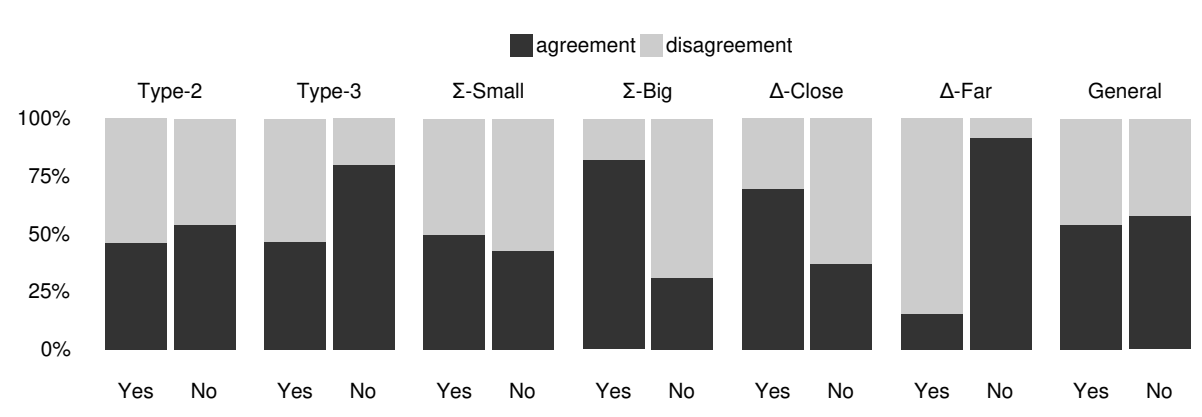

(a) FastR

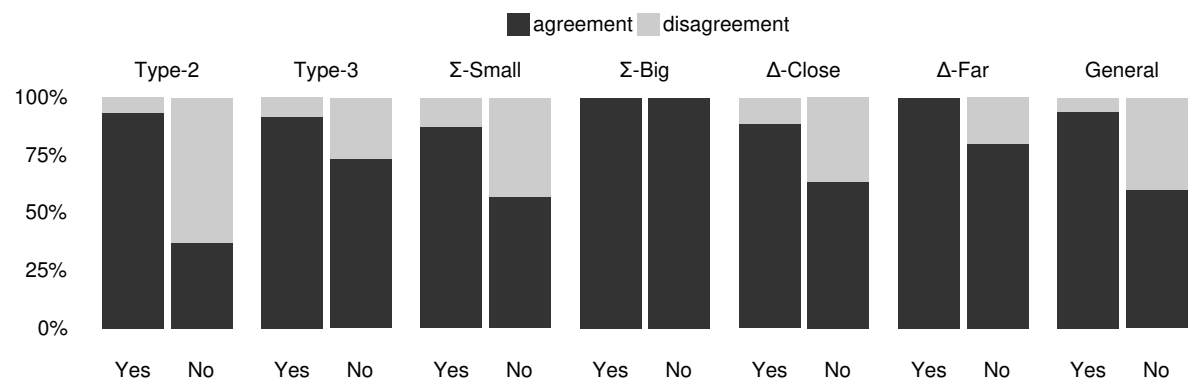

(b) GumTree

Fig. 7 Agreement between the expert and the majority.

Table 6 Cramer's V interpretation.

\begin{tabular}{lc}
\hline Cramer' V & Effect size \\
\hline$\leq 0.10$ & Very weak \\
] $0.10,0.19]$ & Weak \\
] $0.20,0.29]$ & Moderate \\
$\geq 0.30$ & Strong \\
\hline
\end{tabular}

For the type characteristic, the p-value is $0.07(V=0.19)$ for FastR and $0.01(V=0.25)$ for GumTree. The p-value for FastR is not significant using a 0.05 ratio. However, the pvalue for GumTree is significant, and the effect size moderate. It indicates that this characteristic could have an influence in the GumTree project. For the size characteristic, the p-value is $0.04(V=0.20)$ for FastR and 7.1 $e^{-3}(V=0.37)$ for GumTree. The p-value for FastR is significant, and the effect size moderate. The p-value for GumTree is also significant, and the effect size strong. It indicates that this characteristic could have an influence in both projects. Nevertheless, results of this chi-square test are unreliable due to the small number of clones in the $\Sigma$-Big cluster. One can note that Yate's continuity correction has been used because of the small number of clones in the $\Sigma$-Big cluster for GumTree. For the distance characteristic the p-value is $1(V=0)$ for FastR and $0.07(V=0.26)$ for GumTree. Both p-values are not significant. Finally, the Size characteristic is the only one that has an influence on both projects. It is significantly harder for external raters to agree with the expert on small clones. For these clones, using external raters could be unreliable, and thus using an expert of the project seems mandatory. 
Table 7 Number of identical and different answers between the expert and the majority.

\begin{tabular}{lcc}
\hline Project & Identical & Different \\
\hline FastR & 199 & 101 \\
GumTree & 191 & 43 \\
\hline
\end{tabular}

Table 8 Number of identical and different answers between the expert and the majority for true and false positives as judged by the expert.

\begin{tabular}{cccccc}
\hline & \multicolumn{2}{c}{ FastR } & & \multicolumn{2}{c}{ GumTree } \\
\cline { 2 - 3 } \cline { 5 - 6 } Expert judgment & Identical & Different & & Identical & Different \\
\hline Yes & 72 & 61 & & 64 & 4 \\
No & 97 & 70 & & 100 & 66 \\
\hline
\end{tabular}

Project and expert judgment To assess the influence of the project we construct a $2 \times 2$ contingency table with the projects as lines, and as rows the number of identical and different answers in the related project, as shown in table 7 . We use a chi-square test, that yields a $\mathrm{p}$-value of $1.1 e^{-3}(V=0.14)$. This p-value is significant and the effect is weak. It means that it seems significantly harder to judge the clones of FastR than GumTree in the same way as the expert. Therefore, clones of some projects may not be judged by external raters.

To evaluate if the judgment of the expert about clones has an effect on the number of identical answers with the majority of raters, we use the union of clones of all clusters, denoted as general, as explained in section 4.3. We start by investigating if it is easier to agree with a false or true positive as judged by the expert. To that extent we partition all clones into two sets: clones judged positively by the expert (the yes set) and clones judged negatively by the expert (the no set). For each set, we count the number of identical/different answers between the majority and the expert of each project. We use these numbers to build a $2 \times 2$ contingency table as shown in table 8 . Similarly to the previous section, we use a chi-square test that yields a p-value of $0.49(V=0.04)$ for FastR and a p-value of $2.8 e^{-7}(V=0.34)$ for GumTree. The p-value for GumTree is significant. It means that it is significantly harder for external raters to judge the true positives than the false positives in GumTree. Indeed, when judging true positives, external raters give more different answers than identical answers with the expert. On the contrary, when judging false positives, external raters give more identical answers than different ones. This indicates that using external raters to judge true positives could be unreliable. For these clones, using an expert of the project seems mandatory. Some mitigations are required as we do not observe the same effect in FastR. We acknowledge the fact that more projects need to be examined to draw more general conclusions.

\subsection{Participant feedback}

After having conducted the experiment, all the participants have performed a meeting where the important issues concerning the difficulty of rating clones were discussed. These issues are categorized and discussed in the remainder of this section. 


\subsubsection{Clone rating}

Every participant found that it was very difficult to rate the clones in a homogeneous way. Indeed, their judgment evolved as they rated clones (as confirmed in table 2).

Every participant found that it would have been easier to rate the clones if they were ordered by clone classes. At least it would have improved the homogeneity of the answers.

Two participants found that it would have been easier to rate the clones if they were ordered by context (clones within the same function and the same Java class). They explained that it sometimes takes time to understand the surrounding code of a clone, and it would thus be better to make this effort only once.

Every participant noticed that is was easier to decide if a clone is of interest for refactoring purpose than co-evolution purpose. They motivated their answer by explaining that coevolution requires a much deeper knowledge of the code. However, they do not always agree on refactoring opportunities. For instance, some of them would apply refactoring operations for a single line while others require a significant amount of code to perform refactoring.

\subsubsection{Clone detection tool}

Alignment Every participant had trouble with some clones detected by iClones that are not aligned with the structure of the code (for instance a clone that starts in a function and ends in another). This is caused by the fact that iClones is a token-based detection tool. All the participants discarded several clones that could have been useful if better aligned to the code structure.

Extension Every participant also remarked that iClones computed some clones that could have been extended easily. Experts and non-experts had trouble to rate these clones. Each participant judged these clones consistently according to its own strategy. Some decided to keep the clones, while several others decided to discard them.

The participants identified all together 43 clones that could have been extended, 32 for FastR and 11 for GumTree, amounting to $8 \%$ of the rated clones. Results about inter-rater reliability (section 5.2 page 13) have been recalculated from the set of 491 clones $(534-43$ ) corresponding to the ones that do not require to be extended. No significative changes have been observed. Hence, our results are not affected by these clones. As a consequence, during the construction of a clone benchmark, raters should be able to modify the boundaries of a clone candidate before its classification. One can note that Bellon used this good practice in its benchmark construction [3].

To conclude, it seems that a clone detector should both, increase the size of a clone to the maximum according to the code structure, then shorten clones to align their boundary on the boundary of the code. This however requires either a language dependent clone detector tool or at least a post-processing step.

\subsubsection{Programming language}

Two participants noticed that the features of the programming language had sometimes a big impact on the relevance of a clone. For instance, static typing of the Java language makes some clones irrelevant, but these clones would have been of great help in a dynamically typed language. These was particularly emphasized on FastR which requires type inlining due to Java boxing. 
Two participants found that the visitor pattern induces the presence of a lot of clones which are hard to rate.

\section{Threats to validity}

We have identified the following threats to validity of our study.

\subsection{Construct validity}

The main threat to construct validity is related to the process of building the clusters of clones, from which we do sampling. There could be some influence between the clusters, i.e., a big clone can have more odds to be a type- 3 clones. This could bias the result of the influence of the type, size and distance characteristics. However, we did not observe any influence of these characteristics. Similarly, the effect of the expert judgments is based on the general set of clones as explained in section 4.3. Since this set is the union of all the clusters, and not a random sampling of all clones, it may be biased. However, we did show that the distributions of the characteristics of these clones is similar to the ones of all clones.

\subsection{Internal validity}

Our empirical evaluation bears several threats to internal validity. First, raters may talk to each other during the evaluation process, and influence their judgments. Since participants are authors of the paper, they agreed beforehand not to talk about the survey until all have completed it. A second threat is that all the raters are authors of the paper, and this could influence their answers. To minimize this threat, we make all the data collected during the experiments publicly available for examination and replay (see footnote of section 4). Another threat is related to the process of constructing our clusters. We minimize this threat by using a well known clustering algorithm (neural gas) to extract the clusters for each characteristic. This clustering algorithm has been selected because it has the reputation to be very stable (it always finds the same clusters if run multiple times). The number of samples we draw within each cluster may also not be sufficient to be representative in some clusters (especially the one with a huge population such as $\Sigma$-Small or $\Delta$-Close). Unfortunately, manual clone inspection is very expensive and we were limited by the total number of clones a rater can assess. One of the external raters of GumTree has already submitted several patches to this project, and this could have influenced his agreement with the expert. However he was not considering himself as an expert of the project, as his intervention in the code is very limited. Another threat is related to the removal of comments in the two projects under study. Non-experts might have a more difficult time judging the clones. This threat should be addressed in a replication study in order to complete our results. Thus, the impact of comments in such a study could be evaluated.

\subsection{External validity}

Threats to external validity refer to the generalization of our findings. First, we have focused this study on two projects which definitely may not represent all real-world projects. However, the number of projects that can be investigated is limited by the necessity of having one 
expert in the set of raters, and by the number of clones a rater can assess. Additionally, both projects use Java as the main language. Therefore, the results could be different in projects in other programming languages. Nevertheless, Java is recognized as one of the most popular programming languages used in software projects [4]. Moreover, Walenstein et al. also find that system specifics appear to have an impact on inter-rater reliability [28]. Further validation on more projects written in other programming languages should be performed. In this way, we provide all necessary data to replicate this study and complete the findings (see footnote of section 4). A second threat is that we only rely on four raters and the results may change with more raters. Although each selected project has a different expert, our findings may not be generalizable to other open source projects. However, all raters of our study have an extensive Java programming experience and none of them is an undergraduate student. Another threat is related to the use of only one clone detection tool in our study. Once again, the number of clones a rater can assess limits the number of clone detectors that can be considered. Nevertheless, to minimize this threat we rely on both a reputed Type- 3 clone detector, iClones, and the work done by Wang et al. [29] to define the configuration file. The configuration recommended by Wang et al. is such that it maximizes the set of clones that would have been discovered by all main clone detection tools, thus limiting this threat to validity. Additionally, we provide all necessary data to replicate this experiment with other clone detection tools that would be compatible with Java generics. Finally, we investigated only one question about the clones in our study. Our results could change with a different question. However, the question we asked is very representative of the use of code clones by software developers. A last threat is related to the Type- 1 clones we ignored in this experiment. In a previous work [5], we found that developers have a better agreement on Type-1 clones than on other types. Based on this result, we focused on other characteristics, despite no experts were involved in this previous work. Finally, although Type-1 clones should be included to complete the study, our findings are not affected.

\section{Conclusion and future work}

In this article, we investigate reliability of rater judgments about context-dependent clones. We examine two kinds of raters: external raters and experts. We find that external rater judgment may not be reliable. Firstly, external rater answers repeatability seems not good. This indicates that a training session would be beneficial to increase the repeatability of answers. Secondly, the inter-rater reliability seems poor among external raters, and also between external raters and experts. This is a critical issue since it might lead to benchmarks that do not reflect the judgment of project experts, and thus conduct to unreliable contextdependent clone benchmarks. We show that using the majority vote is one way to mitigate this issue. Finally we have seen that there are several characteristics that appears to have a significant effect on the number of identical and different answers between external raters and experts. First of all, the chosen project and expert has a weak to moderate influence on this matter. It means that some projects yield clones that could not be judged by external raters. Second true positive clones seem harder to judge by external raters than false positive clones. Indeed, this characteristic has a strong influence on the number of identical and different answers. Third, it seems harder for external raters to agree with the expert on small clones. Therefore, it seems mandatory to rely on a project expert to validate either true positives or small clones in a benchmark. While the generalization of certain findings is limited, this study is a first and necessary step in the construction of reliable and high quality 
context-dependent clone benchmarks. The availability of all data used and collected during this study is heading in this direction.

As future work, we plan to investigate more clone characteristics and their effect on the agreement (for instance the textual similarity between two fragments of a clone). We also plan to ask other questions than the one we use in our study to assess the impact of the question in the agreement level. Finally we want to use our findings to build high quality benchmarks of clones and to design a machine learning based approach that better eliminates irrelevant clones.

\section{References}

1. Baker, B.S.: On finding duplication and near-duplication in large software systems. In: Proceedings of the Second Working Conference on Reverse Engineering, WCRE '95, pp. 86-. IEEE Computer Society, Washington, DC, USA (1995)

2. Baxter, I., Yahin, A., Moura, L., Sant'Anna, M., Bier, L.: Clone detection using abstract syntax trees. In: Software Maintenance, 1998. Proceedings., International Conference on, pp. 368-377 (1998). DOI 10.1109/ICSM.1998.738528

3. Bellon, S., Koschke, R., Antoniol, G., Krinke, J., Merlo, E.: Comparison and evaluation of clone detection tools. Software Engineering, IEEE Transactions on 33(9), 577-591 (2007). DOI 10.1109/TSE.2007.70725

4. Bissyandé, T.F., Thung, F., Wang, S., Lo, D., Jiang, L., Réveillère, L.: Empirical Evaluation of Bug Linking. In: Proceedings of the 17th European Conference on Software Maintenance and Reengineering (CSMR 2013), pp. 1-10. Genova, Italy (2013)

5. Charpentier, A., Falleri, J.R., Lo, D., Réveillère, L.: An empirical assessment of bellon's clone benchmark. In: Proceedings of the 19th International Conference on Evaluation and Assessment in Software Engineering, EASE '15, pp. 20:1-20:10. ACM, New York, NY, USA (2015). DOI 10.1145/2745802.2745821. URL http://doi.acm.org/10.1145/2745802.2745821

6. Ducasse, S., Rieger, M., Demeyer, S.: A language independent approach for detecting duplicated code. In: Software Maintenance, 1999. (ICSM '99) Proceedings. IEEE International Conference on, pp. 109118 (1999). DOI 10.1109/ICSM.1999.792593

7. Falleri, J.R., Morandat, F., Blanc, X., Martinez, M., Monperrus, M.: Fine-grained and Accurate Source Code Differencing. In: Proceedings of the International Conference on Automated Software Engineering, pp. -. Sweden (2014)

8. Faust, D., Verhoef, C.: Software product line migration and deployment. Software Practice and Experience, John Wiley \& Sons, Ltd 33, 933-955 (2003)

9. Gode, N., Koschke, R.: Incremental clone detection. In: Software Maintenance and Reengineering, 2009. CSMR '09. 13th European Conference on, pp. 219-228 (2009). DOI 10.1109/CSMR.2009.20

10. Ihaka, R., Gentleman, R.: R: a language for data analysis and graphics. Journal of computational and graphical statistics 5(3), 299-314 (1996)

11. Jiang, L., Misherghi, G., Su, Z.: Deckard: Scalable and accurate tree-based detection of code clones. In: In ICSE, pp. 96-105 (2007)

12. Kalibera, T., Maj, P., Morandat, F., Vitek, J.: A fast abstract syntax tree interpreter for R. In: 10th ACM SIGPLAN/SIGOPS International Conference on Virtual Execution Environments, VEE '14, Salt Lake City, UT, USA, March 01 - 02, 2014, pp. 89-102 (2014). DOI 10.1145/2576195.2576205

13. Kamiya, T., Kusumoto, S., Inoue, K.: Ccfinder: a multilinguistic token-based code clone detection system for large scale source code. Software Engineering, IEEE Transactions on 28(7), 654-670 (2002). DOI 10.1109/TSE.2002.1019480

14. Kapser, C., Anderson, P., Godfrey, M., Koschke, R., Rieger, M., van Rysselberghe, F., Weï̈gerber, P.: Subjectivity in clone judgment: Can we ever agree? In: Duplication, Redundancy, and Similarity in Software, no. 06301 in Dagstuhl Seminar Proceedings. IBFI, Schloss Dagstuhl, Germany (2006)

15. Kapser, C., Godfrey, M.: "cloning considered harmful" considered harmful. In: Reverse Engineering, 2006. WCRE '06. 13th Working Conference on, pp. 19-28 (2006). DOI 10.1109/WCRE.2006.1

16. Koschke, R., Falke, R., Frenzel, P.: Clone detection using abstract syntax suffix trees. In: Proceedings of the 13th Working Conference on Reverse Engineering, WCRE '06, pp. 253-262. IEEE Computer Society, Washington, DC, USA (2006). DOI 10.1109/WCRE.2006.18. URL http://dx.doi.org/10.1109/WCRE.2006.18 
17. Krutz, D.E., Le, W.: A code clone oracle. In: Proceedings of the 11th Working Conference on Mining Software Repositories, MSR 2014, pp. 388-391. ACM, New York, NY, USA (2014). DOI 10.1145/2597073.2597127. URL http://doi.acm.org.gate6.inist.fr/10.1145/2597073.2597127

18. Lague, B., Proulx, D., Mayrand, J., Merlo, E.M., Hudepohl, J.: Assessing the benefits of incorporating function clone detection in a development process. In: Proceedings of the International Conference on Software Maintenance, ICSM '97, pp. 314-. IEEE Computer Society, Washington, DC, USA (1997). URL http://dl.acm.org/citation.cfm?id=645545.853273

19. Landis, J.R., Koch, G.G.: The measurement of observer agreement for categorical data. biometrics pp. 159-174 (1977)

20. Li, Z., Lu, S., Myagmar, S., Zhou, Y.: Cp-miner: finding copy-paste and related bugs in largescale software code. Software Engineering, IEEE Transactions on 32(3), 176-192 (2006). DOI 10.1109/TSE.2006.28

21. Martinetz, T., Schulten, K.: A "Neural-Gas" Network Learns Topologies. In: Artificial Neural Networks, vol. I, pp. 397-402 (1991)

22. Mende, T., Koschke, R., Beckwermert, F.: An evaluation of code similarity identification for the growand-prune model. Journal on Software Maintenance and Evolution 21(2), 143-169 (2009)

23. Nguyen, H.A., Nguyen, T.T., Pham, N., Al-Kofahi, J., Nguyen, T.: Clone management for evolving software. Software Engineering, IEEE Transactions on 38(5), 1008-1026 (2012). DOI 10.1109/TSE.2011.90

24. Roy, C., Cordy, J.: Nicad: Accurate detection of near-miss intentional clones using flexible prettyprinting and code normalization. In: Program Comprehension, 2008. ICPC 2008. The 16th IEEE International Conference on, pp. 172-181 (2008). DOI 10.1109/ICPC.2008.41

25. Roy, C., Cordy, J.: A mutation/injection-based automatic framework for evaluating code clone detection tools. In: Software Testing, Verification and Validation Workshops, 2009. ICSTW '09. International Conference on, pp. 157-166 (2009). DOI 10.1109/ICSTW.2009.18

26. Selim, G., Foo, K., Zou, Y.: Enhancing source-based clone detection using intermediate representation. In: Reverse Engineering (WCRE), 2010 17th Working Conference on, pp. 227-236 (2010). DOI 10.1109/WCRE.2010.33

27. Svajlenko, J., Islam, J.F., Keivanloo, I., Roy, C.K., Mia, M.M.: Towards a big data curated benchmark of inter-project code clones. ICSME p. 5 (2014)

28. Walenstein, A., Jyoti, N., Li, J., Yang, Y., Lakhotia, A.: Problems creating task-relevant clone detection reference data. In: Reverse Engineering, 2003. WCRE 2003. Proceedings. 10th Working Conference on, pp. 285-294 (2003). DOI 10.1109/WCRE.2003.1287259

29. Wang, T., Harman, M., Jia, Y., Krinke, J.: Searching for better configurations: A rigorous approach to clone evaluation. In: Proceedings of the 2013 9th Joint Meeting on Foundations of Software Engineering, ESEC/FSE 2013, pp. 455-465. ACM, New York, NY, USA (2013). DOI 10.1145/2491411.2491420

30. Yang, J., Hotta, K., Higo, Y., Igaki, H., Kusumoto, S.: Classification model for code clones based on machine learning. Empirical Software Engineering pp. 1-31 (2014) 